PROCEEDINGS OF THE

AMERICAN MATHEMATICAL SOCIETY

Volume 131, Number 9, Pages 2895-2902

S 0002-9939(03)06932-6

Article electronically published on January 28, 2003

\title{
ARBITRARILY LARGE SOLUTIONS OF THE CONFORMAL SCALAR CURVATURE PROBLEM AT AN ISOLATED SINGULARITY
}

\author{
STEVEN D. TALIAFERRO AND LEI ZHANG
}

(Communicated by Bennett Chow)

ABSTRACT. We study the conformal scalar curvature problem

$$
k(x) u^{\frac{n+2}{n-2}} \leq-\Delta u \leq u^{\frac{n+2}{n-2}} \quad \text { in } \quad \mathbf{R}^{n}, n \geq 3,
$$

where $k: \mathbf{R}^{n} \rightarrow(0,1]$ is a continuous function. We show that a necessary and sufficient condition on $k$ for this problem to have $C^{2}$ positive solutions which are arbitrarily large at $\infty$ is that $k$ be less than 1 on a sequence of points in $\mathbf{R}^{n}$ which tends to $\infty$.

\section{INTRODUCTION}

In this paper we study the conformal scalar curvature problem

$$
k(x) u^{n^{*}} \leq-\Delta u \leq u^{n^{*}} \quad \text { in } \quad \mathbf{R}^{n}, n \geq 3,
$$

where $n^{*}=(n+2) /(n-2)$. We also study the problem

$$
k(x) u^{n^{*}} \leq-\Delta u \leq K(x) u^{n^{*}} \quad \text { in } \quad \mathbf{B}^{n}-\{0\}, n \geq 3,
$$

where $\mathbf{B}^{n}=\left\{x \in \mathbf{R}^{n}:|x|<1\right\}$.

The following theorem gives conditions on $k$ under which (1.1) has $C^{2}$ positive solutions which are arbitrarily large at $\infty$.

Theorem 1. Let $k: \mathbf{R}^{n} \rightarrow(0,1]$ be a continuous function such that for some sequence $\left\{x^{j}\right\}_{j=1}^{\infty}$ of distinct points in $\mathbf{R}^{n}$ satisfying $\lim _{j \rightarrow \infty}\left|x^{j}\right|=\infty$ we have

$$
k\left(x^{j}\right)<1 \quad \text { for } \quad j=1,2, \ldots
$$

Then, for each continuous function $\varphi:(1, \infty) \rightarrow(0, \infty)$, there exists a $C^{2}$ positive solution $u(x)$ of (1.1) satisfying

$$
u(x) \neq \mathcal{O}(\varphi(|x|)) \quad \text { as } \quad|x| \rightarrow \infty .
$$

The following corollary of Theorem 1 gives necessary and sufficient conditions on the function $k$ for $C^{2}$ positive solutions of (1.1) to satisfy an a priori bound at $\infty$.

Received by the editors March 1, 2002 and, in revised form, April 11, 2002.

2000 Mathematics Subject Classification. Primary 35J60, 53C21.

(C)2003 American Mathematical Society 
Corollary 1. Let $k: \mathbf{R}^{n} \rightarrow(0,1]$ be a continuous function. Then there exists a continuous function $\varphi:(1, \infty) \rightarrow(0, \infty)$ such that each $C^{2}$ positive solution $u(x)$ of (1.1) satisfies

$$
u(x)=\mathcal{O}(\varphi(|x|)) \quad \text { as } \quad|x| \rightarrow \infty
$$

if and only if $k$ is identically equal to 1 in the complement of some compact subset of $\mathbf{R}^{n}$. In this case, (1.3) holds with $\varphi(r)=r^{-(n-2) / 2}$.

Proof. The "only if" part of Corollary 1 follows from Theorem 1. The "if" part was proved by Caffarelli, Gidas, and Spruck [1].

The next corollary of Theorem 1 deals with the case the isolated singularity of (1.1) is at the origin instead of at $\infty$.

Corollary 2. Let $\kappa:\left(\mathbf{R}^{n}-\{0\}\right) \rightarrow(0,1]$ be a continuous function such that for some sequence $\left\{y^{j}\right\}_{j=1}^{\infty}$ of distinct points in $\mathbf{R}^{n}-\{0\}$ satisfying $\lim _{j \rightarrow \infty}\left|y^{j}\right|=0$ we have

$$
\kappa\left(y^{j}\right)<1 \quad \text { for } \quad j=1,2, \ldots
$$

Then, for each continuous function $\varphi:(0,1) \rightarrow(0, \infty)$, there exists a $C^{2}$ positive solution $v(y)$ of

$$
\begin{array}{r}
\kappa(y) v^{n^{*}} \leq-\Delta v \leq v^{n^{*}} \quad \text { in } \quad \mathbf{R}^{n}-\{0\}, n \geq 3, \\
\lim _{|y| \rightarrow \infty}|y|^{n-2} v(y)=L \quad \text { for some } L \in(0, \infty)
\end{array}
$$

satisfying

$$
v(y) \neq \mathcal{O}(\varphi(|y|)) \quad \text { as } \quad|y| \rightarrow 0^{+} .
$$

Proof. Clearly there exists a continuous function $\hat{\kappa}:\left(\mathbf{R}^{n}-\{0\}\right) \rightarrow(0,1]$ such that $\kappa \leq \hat{\kappa}$ in $\mathbf{R}^{n}-\{0\}, \hat{\kappa}\left(y^{j}\right)<1$ for $j=1,2, \ldots$, and $\hat{\kappa}$ is identically equal to 1 in the complement of some compact subset of $\mathbf{R}^{n}$. It therefore suffices to prove Corollary 2 under the assumption that $\kappa$ is identically equal to 1 in the complement of some compact subset of $\mathbf{R}^{n}$. Define $k: \mathbf{R}^{n} \rightarrow(0,1]$ by $k(0)=1$ and $k(x)=\kappa\left(x /|x|^{2}\right)$ for $x \in \mathbf{R}^{n}-\{0\}$. Then $k$ is continuous and the Kelvin transform, $v(y)=|x|^{n-2} u(x)$, $x=y /|y|^{2}$, of each $C^{2}$ positive solution $u(x)$ of (1.1) is a $C^{2}$ positive solution of (1.4) with $L=u(0)$. Thus Corollary 2 follows from Theorem 1.

The following theorem gives conditions on $k$ and $K$ under which (1.2) has $C^{2}$ positive solutions which are arbitrarily large at the origin.

Theorem 2. Let $k, K:\left(\mathbf{B}^{n}-\{0\}\right) \rightarrow \mathbf{R}$ be continuous functions such that for some positive constants $a$ and $b$ we have

$$
a \leq k \leq K \leq b \quad \text { in } \quad \mathbf{B}^{n}-\{0\}
$$

and for some sequence $\left\{x^{j}\right\}_{j=1}^{\infty}$ of distinct points in $\mathbf{B}^{n}-\{0\}$ satisfying $\lim _{j \rightarrow \infty}\left|x^{j}\right|$ $=0$ we have

$$
k\left(x^{j}\right)<K\left(x^{j}\right) \quad \text { for } \quad j=1,2, \ldots
$$

Then, for each continuous function $\varphi:(0,1) \rightarrow(0, \infty)$, there exists a $C^{1}$ positive solution $u(x)$ of (1.2) satisfying

$$
u(x) \neq \mathcal{O}(\varphi(|x|)) \quad \text { as } \quad|x| \rightarrow 0^{+} .
$$

If, in addition, either $k$ or $K$ is locally Hölder continuous with exponent $\alpha$ in $\mathbf{B}^{n}-$ $\{0\}$ for some $\alpha \in(0,1)$, then, for each continuous function $\varphi:(0,1) \rightarrow(0, \infty)$, there exists a $C^{2}$ positive solution $u(x)$ of (1.2) satisfying (1.5). 
In light of Corollary 2 and Theorem 2, it is natural to ask the

Open question. Is Theorem 2 true in $\mathbf{R}^{n}-\{0\}$ ?

By ingeniously piecing together modified Delaunay-Fowler type solutions, Leung [4] very recently proved, for each positive constant $\varepsilon$ and each continuous function $\varphi:(0,1) \rightarrow(0, \infty)$, that the problem

$$
(1-\varepsilon) u^{n^{*}} \leq-\Delta u \leq u^{n^{*}} \quad \text { in } \quad \mathbf{R}^{n}-\{0\}, n \geq 3,
$$

has a $C^{2}$ positive solution $u(x)$ satisfying (1.5). Our method of proving Theorem 1 , consisting of piecing together the simpler standard bubbles (see Section 2), is very different than his, and our proof is significantly shorter. Leung also shows that there exists a positive Lipschitz continuous function $K(x)$ on $\mathbf{R}^{n}$ such that the equation

$$
-\Delta u=K(x) u^{n^{*}} \quad \text { in } \quad \mathbf{R}^{n}-\{0\}, n>4,
$$

has a $C^{2}$ positive solution $u(x)$ not satisfying

$$
u(x)=\mathcal{O}\left(|x|^{-(n-2) / 2}\right) \quad \text { as } \quad|x| \rightarrow 0^{+} .
$$

In the other direction, Korevaar, Mazzeo, Pacard, and Schoen 3 obtain precise asymptotic estimates at the origin for singular positive solutions of

$$
-\Delta u=K(x) u^{n^{*}} \quad \text { in } \quad \mathbf{B}^{n}-\{0\}, n \geq 3,
$$

when $K(x) \equiv 1$. In the case of non-constant $K$, Chen and Lin [2], 5] and Zhang 9] give conditions on $K$ such that every $C^{2}$ positive solution $u(x)$ of (1.7) satisfies (1.6).

Finally, Taliaferro [7], [8] has studied arbitrarily large solutions of (1.1) with the exponent $n^{*}$ replaced by a constant $\lambda<n^{*}$.

\section{Proofs}

In this section we prove Theorems 1 and 2 . But first we introduce some notation and state some easily verified facts that will be used in these proofs.

Let

$$
w(r, \sigma)=\frac{[n(n-2)]^{\frac{n-2}{4}} \sigma^{\frac{n-2}{2}}}{\left(\sigma^{2}+r^{2}\right)^{\frac{n-2}{2}}} .
$$

It is well-known that the function $U(x):=w(|x|, \sigma)$, which is sometimes called a bubble, satisfies $-\Delta U=U^{n^{*}}$ in $\mathbf{R}^{n}$ for each positive constant $\sigma$. Thus letting

$$
\rho(x)=w(|x|, 1) / 2^{n / 2}
$$

we have

$$
-\Delta \rho=2^{n^{*}+1} \rho^{n^{*}} \quad \text { in } \quad \mathbf{R}^{n} .
$$

As $\sigma \rightarrow 0^{+}, w(|x|, \sigma)$ and each of its partial derivatives with respect to $x$ converges uniformly to zero on each closed subset of $\mathbf{R}^{n}-\{0\}$.

Define $f:[0, \infty) \times(0,1) \times(0, \infty) \rightarrow \mathbf{R}$ and $M:(0,1) \times(0, \infty) \rightarrow \mathbf{R}$ by

$$
f(z, \psi, \zeta)=\psi(\zeta+z)^{n^{*}}-z^{n^{*}} \quad \text { and } \quad M(\psi, \zeta)=\frac{\psi \zeta^{n^{*}}}{\left(1-\psi^{\frac{1}{n^{*}-1}}\right)^{n^{*}-1}} .
$$


For each fixed $(\psi, \zeta) \in(0,1) \times(0, \infty)$, the function $f(\cdot, \psi, \zeta):[0, \infty) \rightarrow \mathbf{R}$ assumes its maximum at

$$
z_{(\psi, \zeta)}=\frac{\zeta \psi^{\frac{1}{n^{*}-1}}}{1-\psi^{\frac{1}{n^{*}-1}}} \quad \text { and } \quad f\left(z_{(\psi, \zeta)}, \psi, \zeta\right)=M(\psi, \zeta) .
$$

Let $N$ be the Newtonian potential operator over $\mathbf{R}^{n}$ defined by

$$
(N g)(x)=\frac{1}{(n-2) n \omega_{n}} \int_{\mathbf{R}^{n}} \frac{1}{|x-y|^{n-2}} g(y) d y
$$

where $\omega_{n}$ is the volume of $\mathbf{B}^{n}$.

Proof of Theorem 1. Clearly there exists a $C^{\infty}$ function $\hat{k}: \mathbf{R}^{n} \rightarrow(0,1]$ such that $k \leq \hat{k}$ in $\mathbf{R}^{n}$ and $\hat{k}\left(x^{j}\right)<1$ for $j=1,2, \ldots$ It therefore suffices to prove Theorem 1 under the assumption that $k$ is $C^{\infty}$ in $\mathbf{R}^{n}$.

Choose a sequence $\left\{r_{j}\right\}_{j=1}^{\infty} \subset(0,1)$ such that

$$
B_{2 r_{i}}\left(x^{i}\right) \cap B_{2 r_{j}}\left(x^{j}\right)=\emptyset \quad \text { for } \quad i \neq j
$$

and $k<1$ in $\overline{B_{r_{j}}\left(x^{j}\right)}$. Since $M(k(x), 2 \rho(x))$ is bounded in each of the balls $B_{r_{j}}\left(x^{j}\right)$ (but not in their union), by sufficiently decreasing each $r_{j}$, we can force the function

$$
\hat{M}:= \begin{cases}M_{j}:=\sup _{B_{r_{j}}\left(x^{j}\right)} M(k(x), 2 \rho(x)) & \text { in } B_{r_{j}}\left(x^{j}\right), \\ 0 & \text { in } \mathbf{R}^{n}-\bigcup_{j=1}^{\infty} B_{2 r_{j}}\left(x^{j}\right), \\ \left(2-\left|x-x^{j}\right| / r_{j}\right) M_{j} & \text { in } B_{2 r_{j}}\left(x^{j}\right)-B_{r_{j}}\left(x^{j}\right)\end{cases}
$$

to satisfy

$$
N \hat{M}<\frac{1}{2} \rho \quad \text { in } \quad \mathbf{R}^{n} .
$$

(More precisely, we can force $\hat{M}$ to satisfy $(2.2)$ by choosing $r_{j}$ positive and so small that the Newtonian potential of the characteristic function of $B_{2 r_{j}}\left(x^{j}\right)$ is less than $\rho /\left(2^{j+1} M_{j}\right)$ in $\mathbf{R}^{n}$. This is possible because $\rho$ is positive and decays at $\infty$ like $|x|^{2-n}$.) Since $\hat{M}$ is locally Lipschitz continuous in $\mathbf{R}^{n}$ we have $\bar{v}:=\rho / 2+N \hat{M} \in$ $C^{2}\left(\mathbf{R}^{n}\right)$ and

$$
-\Delta \bar{v}=2^{n^{*}} \rho^{n^{*}}+\hat{M} \quad \text { in } \quad \mathbf{R}^{n}
$$

by (2.1). It follows from (2.2) that

$$
\frac{1}{2} \rho<\bar{v}<\rho \quad \text { in } \quad \mathbf{R}^{n} .
$$

Let $\varepsilon_{j}$ be a sequence of positive numbers such that $\sum_{j=1}^{\infty} \varepsilon_{j}=1$. For each positive integer $j$, choose $\sigma_{j}$ positive and so small that $u_{j}(x):=w\left(\left|x-x^{j}\right|, \sigma_{j}\right)$ satisfies

$$
\begin{aligned}
& u_{j}<\varepsilon_{j} \rho \quad \text { in } \quad \mathbf{R}^{n}-B_{r_{j}}\left(x^{j}\right), \\
& u_{j}\left(x^{j}\right)>j \varphi\left(\left|x^{j}\right|\right),
\end{aligned}
$$

$\sum_{j=1}^{\infty} u_{j} \in C^{\infty}\left(\mathbf{R}^{n}\right)$, and

$$
-\Delta\left(\sum_{j=1}^{\infty} u_{j}\right)=\sum_{j=1}^{\infty} u_{j}^{n^{*}} \quad \text { in } \quad \mathbf{R}^{n}
$$


Define $\underline{H}, H: \mathbf{R}^{n} \times[0, \infty) \rightarrow \mathbf{R}$ by

$$
\begin{aligned}
& \underline{H}(x, v)=k(x)\left(v+\sum_{j=1}^{\infty} u_{j}(x)\right)^{n^{*}}-\sum_{j=1}^{\infty} u_{j}(x)^{n^{*}}, \\
& H(x, v)=\max \{0, \underline{H}(x, v)\} .
\end{aligned}
$$

For $x \in B_{r_{j}}\left(x^{j}\right)$ and $0 \leq v \leq \rho(x)$ it follows from (2.5) that

$$
\begin{aligned}
\underline{H}(x, v) & <k(x)\left(v+u_{j}(x)+\sum_{i \neq j} u_{i}(x)\right)^{n^{*}}-u_{j}(x)^{n^{*}} \\
& <k(x)\left(2 \rho(x)+u_{j}(x)\right)^{n^{*}}-u_{j}(x)^{n^{*}} \\
& =f\left(u_{j}(x), k(x), 2 \rho(x)\right) \\
& \leq M(k(x), 2 \rho(x)) \leq \hat{M}(x) \leq-(\Delta \bar{v})(x)
\end{aligned}
$$

by (2.3). Also, for $x \in \mathbf{R}^{n}-\bigcup_{j=1}^{\infty} B_{r_{j}}\left(x^{j}\right)$ and $0 \leq v \leq \rho(x)$ we have

$$
\underline{H}(x, v)<k(x)(2 \rho(x))^{n^{*}} \leq-(\Delta \bar{v})(x) .
$$

Hence

$$
0 \leq H(x, v)<-(\Delta \bar{v})(x) \quad \text { for } \quad x \in \mathbf{R}^{n} \text { and } 0 \leq v \leq \rho(x)
$$

because (2.3) implies that $-\Delta \bar{v}>0$ in $\mathbf{R}^{n}$.

Thus by (2.4), for each positive integer $i$ we can use $\underline{v}: \equiv 0$ and $\bar{v}$ as sub and super solutions of the problem

$$
\begin{array}{cc}
-\Delta v=H(x, v) & \text { in } B_{i}(0), \\
v=0 & \text { on } \partial B_{i}(0)
\end{array}
$$

to conclude that this problem has a $C^{2}$ solution $v_{i}$ satisfying $0 \leq v_{i} \leq \rho$. It follows from standard elliptic theory that some subsequence of $v_{i}$ converges to a $C^{2}$ solution $u_{0}$ of

$$
\left.\begin{array}{c}
-\Delta u_{0}=H\left(x, u_{0}\right) \\
0 \leq u_{0} \leq \rho
\end{array}\right\} \quad \text { in } \quad \mathbf{R}^{n}
$$

Clearly $\underline{H} \leq H \leq \bar{H}$ in $\mathbf{R}^{n} \times[0, \infty)$ where

$$
\bar{H}(x, v)=\left(v+\sum_{j=1}^{\infty} u_{j}(x)\right)^{n^{*}}-\sum_{j=1}^{\infty} u_{j}(x)^{n^{*}}
$$

and thus

$$
\underline{H}\left(x, u_{0}(x)\right) \leq H\left(x, u_{0}(x)\right) \leq \bar{H}\left(x, u_{0}(x)\right) \quad \text { in } \quad \mathbf{R}^{n}
$$

which together with (2.7) and (2.8) implies that $u:=\sum_{j=0}^{\infty} u_{j}$ is a $C^{2}$ positive solution of (1.1). Hence Theorem 1 follows from (2.6).

Proof of Theorem 2. By scaling $u$ we see that it suffices to prove Theorem 2 under the assumption that $b=1$. 
Choose a sequence $\left\{r_{j}\right\}_{j=1}^{\infty} \subset(0,1)$ such that $B_{2 r_{j}}\left(x^{j}\right) \subset \mathbf{B}^{n}-\{0\}$,

$$
B_{2 r_{i}}\left(x^{i}\right) \cap B_{2 r_{j}}\left(x^{j}\right)=\emptyset \quad \text { for } \quad i \neq j,
$$

and $k(x) / K_{j}<1$ in $\overline{B_{r_{j}}\left(x^{j}\right)}$, where $K_{j}=\inf _{B_{r_{j}}\left(x^{j}\right)} K$. Since $M\left(k(x) / K_{j}, 2 \rho(x)\right)$ is bounded in each of the balls $B_{r_{j}}\left(x^{j}\right)$ (but not in their union), by sufficiently decreasing each $r_{j}$, we can force the function

$$
\hat{M}:= \begin{cases}M_{j}:=\sup _{B_{r_{j}}\left(x^{j}\right)} M\left(k(x) / K_{j}, 2 \rho(x)\right) & \text { in } B_{r_{j}}\left(x^{j}\right), \\ 0 & \text { in } \mathbf{R}^{n}-\bigcup_{j=1}^{\infty} B_{2 r_{j}}\left(x^{j}\right), \\ \left(2-\left|x-x^{j}\right| / r_{j}\right) M_{j} & \text { in } B_{2 r_{j}}\left(x^{j}\right)-B_{r_{j}}\left(x^{j}\right)\end{cases}
$$

to satisfy

$$
N \hat{M}<\frac{1}{2} \rho \quad \text { in } \quad \mathbf{R}^{n} .
$$

(See the parenthetical remark after equation (2.2).) Since $\hat{M}$ is locally Lipschitz continuous in $\mathbf{R}^{n}-\{0\}$ we have $\bar{v}:=\rho / 2+N \hat{M} \in C^{2}\left(\mathbf{R}^{n}-\{0\}\right)$ and

$$
-\Delta \bar{v}=2^{n^{*}} \rho^{n^{*}}+\hat{M} \quad \text { in } \quad \mathbf{R}^{n}-\{0\}
$$

by (2.1). It follows from (2.9) that

$$
\frac{1}{2} \rho<\bar{v}<\rho \quad \text { in } \quad \mathbf{R}^{n} .
$$

Let

$$
\underline{v}(x) \equiv \beta:=\inf _{\mathbf{B}^{n}} \bar{v} \quad \text { in } \quad \mathbf{B}^{n} .
$$

Clearly $\beta>0$ and later $\underline{v}$ and $\bar{v}$ will be used as sub and super solutions.

Let $\varepsilon_{j}$ be a sequence of positive numbers such that $\sum_{j=1}^{\infty} \varepsilon_{j}=a$. For each positive integer $j$, choose $\sigma_{j}$ positive and so small that $u_{j}(x):=w\left(\left|x-x^{j}\right|, \sigma_{j}\right) / K_{j}^{1 /\left(n^{*}-1\right)}$ satisfies

$$
\begin{aligned}
& u_{j}<\beta \varepsilon_{j} \quad \text { in } \quad \mathbf{R}^{n}-B_{r_{j}}\left(x^{j}\right), \\
& u_{j}\left(x^{j}\right)>j \varphi\left(\left|x^{j}\right|\right),
\end{aligned}
$$

$\sum_{j=1}^{\infty} u_{j} \in C^{\infty}\left(\mathbf{R}^{n}-\{0\}\right)$, and

$$
-\Delta\left(\sum_{j=1}^{\infty} u_{j}\right)=\sum_{j=1}^{\infty} K_{j} u_{j}^{n^{*}} \quad \text { in } \quad \mathbf{R}^{n}-\{0\} .
$$

Define $\underline{H}, H:\left(\mathbf{B}^{n}-\{0\}\right) \times[\beta, \infty) \rightarrow \mathbf{R}$ by

$$
\begin{aligned}
& \underline{H}(x, v)=k(x)\left(v+\sum_{j=1}^{\infty} u_{j}(x)\right)^{n^{*}}-\sum_{j=1}^{\infty} K_{j} u_{j}(x)^{n^{*}}, \\
& H(x, v)=\max \{0, \underline{H}(x, v)\} .
\end{aligned}
$$


For $x \in B_{r_{j}}\left(x^{j}\right)$ and $\beta \leq v \leq \rho(x)$ it follows from (2.12) that

$$
\begin{aligned}
\underline{H}(x, v) & <k(x)\left(v+u_{j}(x)+\sum_{i \neq j} u_{i}(x)\right)^{n^{*}}-K_{j} u_{j}(x)^{n^{*}} \\
& \leq k(x)\left(2 \rho(x)+u_{j}(x)\right)^{n^{*}}-K_{j} u_{j}(x)^{n^{*}} \\
& =K_{j} f\left(u_{j}(x), k(x) / K_{j}, 2 \rho(x)\right) \\
& \leq K_{j} M\left(k(x) / K_{j}, 2 \rho(x)\right) \leq \hat{M}(x) \leq-(\Delta \bar{v})(x)
\end{aligned}
$$

by (2.10). Also, for $x \in\left(\mathbf{B}^{n}-\{0\}\right)-\bigcup_{j=1}^{\infty} B_{r_{j}}\left(x^{j}\right)$ and $\beta \leq v \leq \rho(x)$, we have

$$
\underline{H}(x, v)<k(x)(2 \rho(x))^{n^{*}} \leq-(\Delta \bar{v})(x) .
$$

Hence

$$
0 \leq H(x, v)<-(\Delta \bar{v})(x) \quad \text { for } \quad x \in \mathbf{B}^{n}-\{0\} \text { and } \beta \leq v \leq \rho(x)
$$

because (2.10) implies that $-\Delta \bar{v}>0$ in $\mathbf{R}^{n}-\{0\}$.

Thus by (2.11), for each integer $i>2$ we can use $\underline{v}$ and $\bar{v}$ as sub and super solutions of the problem

$$
\begin{array}{cc}
-\Delta v=H(x, v) & \text { in } \frac{1}{i}<|x|<1-\frac{1}{i}, \\
v=\beta & \text { for }|x|=\frac{1}{i} \text { or }|x|=1-\frac{1}{i}
\end{array}
$$

to conclude that this problem has a $C^{1}$ solution $v_{i}$ satisfying $\beta \leq v_{i} \leq \rho$. It follows from standard elliptic theory that some subsequence of $v_{i}$ converges to a $C^{1}$ solution $u_{0}$ of

$$
\left.\begin{array}{c}
-\Delta u_{0}=H\left(x, u_{0}\right) \\
\beta \leq u_{0} \leq \rho
\end{array}\right\} \quad \text { in } \quad \mathbf{B}^{n}-\{0\}
$$

Defining $\bar{H}:\left(\mathbf{B}^{n}-\{0\}\right) \times[\beta, \infty) \rightarrow \mathbf{R}$ by

$$
\bar{H}(x, v)=K(x)\left(v+\sum_{j=1}^{\infty} u_{j}(x)\right)^{n^{*}}-\sum_{j=1}^{\infty} K_{j} u_{j}(x)^{n^{*}}
$$

we have for $v \geq \beta$ and $x \in\left(\mathbf{B}^{n}-\{0\}\right)-\bigcup_{j=1}^{\infty} B_{r_{j}}\left(x^{j}\right)$ that

$$
\begin{aligned}
\bar{H}(x, v) & \geq a \beta^{n^{*}}-\sum_{j=1}^{\infty}\left(\varepsilon_{j} \beta\right)^{n^{*}} \\
& =\beta^{n^{*}}\left(a-\sum_{j=1}^{\infty} \varepsilon_{j}^{n^{*}}\right)>0
\end{aligned}
$$


and we have for $v \geq \beta$ and $x \in B_{r_{j}}\left(x^{j}\right)$ that

$$
\begin{aligned}
\bar{H}(x, v) & \geq K_{j}\left(\beta+u_{j}(x)\right)^{n^{*}}-K_{j} u_{j}(x)^{n^{*}}-\sum_{i \neq j}^{\infty}\left(\beta \varepsilon_{i}\right)^{n^{*}} \\
& \geq K_{j} \beta^{n^{*}}-\beta^{n^{*}} \sum_{i \neq j}^{\infty} \varepsilon_{i}^{n^{*}} \\
& \geq \beta^{n^{*}}\left(a-\sum_{j=1}^{\infty} \varepsilon_{j}^{n^{*}}\right)>0 .
\end{aligned}
$$

Therefore $\bar{H}$ is a positive function and hence $\underline{H} \leq H \leq \bar{H}$ in $\left(\mathbf{B}^{n}-\{0\}\right) \times[\beta, \infty)$. Thus

$$
\underline{H}\left(x, u_{0}(x)\right) \leq H\left(x, u_{0}(x)\right) \leq \bar{H}\left(x, u_{0}(x)\right) \quad \text { in } \quad \mathbf{B}^{n}-\{0\}
$$

which together with (2.14) and (2.15) implies that $u:=\sum_{j=0}^{\infty} u_{j}$ is a $C^{1}$ positive solution of (1.2). Hence Theorem 2, except for its last sentence, follows from (2.13).

The last sentence of Theorem 2 follows from the following two observations:

(i) If $k \in C^{\alpha}\left(\mathbf{B}^{n}-\{0\}\right)$, then a $C^{1}$ solution $u_{0}$ of (2.15) is necessarily a $C^{2}$ solution.

(ii) If $K \in C^{\alpha}\left(\mathbf{B}^{n}-\{0\}\right)$, then there exists a function $\hat{k} \in C^{\alpha}\left(\mathbf{B}^{n}-\{0\}\right)$ satisfying $k \leq \hat{k} \leq K$ in $\mathbf{B}^{n}-\{0\}$ and $\hat{k}\left(x^{j}\right)<K\left(x^{j}\right)$ for $j=1,2, \ldots$

\section{REFERENCES}

[1] L. A. Caffarelli, B. Gidas, and J. Spruck, Asymptotic symmetry and local behavior of semilinear elliptic equations with critical Sobolev growth, Comm. Pure Appl. Math. 42 (1989), 271-297. MR 90c:35075

[2] C.-C. Chen and C.-S. Lin, Estimates of the scalar curvature equation via the method of moving planes, Comm. Pure Appl. Math. 50 (1997), 971-1017. MR 98k:35051

[3] N. Korevaar, R. Mazzeo, F. Pacard, and R. Schoen, Refined asymptotics for constant scalar curvature metrics with isolated singularities, Invent. Math. 135 (1999), 233-272. MR 2001a:35055

[4] M.-C. Leung, Blow-up solutions of nonlinear elliptic equations in $\mathbf{R}^{n}$ with critical exponent, preprint.

[5] C.-S. Lin, Estimates of the scalar curvature equation via the method of moving planes III, Comm. Pure Appl. Math. 53 (2000), 611-646. MR 2001i:53056

[6] S. D. Taliaferro, On the growth of superharmonic functions near an isolated singularity I, $J$. Differential Equations 158 (1999), 28-47. MR 2000j:35288

[7] S. D. Taliaferro, Isolated singularities of nonlinear elliptic inequalities, Indiana Univ. Math. J. 50 (2001), 1885-1897.

[8] S. D. Taliaferro, Local behavior and global existence of positive solutions of $a u^{\lambda} \leq-\Delta u \leq u^{\lambda}$, Ann. Inst. H. Poincare Anal. Non Lineaire, in press, see http://www.math.tamu.edu/ steven.taliaferro/selpubs.html.

[9] Lei Zhang, Refined asymptotic estimates for conformal scalar curvature equation via moving sphere method, J. Functional Analysis, in press.

Department of Mathematics, Texas A\&M University, College Station, Texas 778433368

E-mail address: stalia@math.tamu.edu

Department of Mathematics, Texas A\&M University, College Station, Texas 778433368

E-mail address: lzhang@math.tamu.edu 